\title{
Formation of Periodic Micro/nano-holes Array in Boro-aluminosilicate Glass by Single-pulse Femtosecond Laser Machining
}

\author{
Md. Shamim AHSAN ${ }^{* 1}$, Yoon-Young KWON ${ }^{* 2}$, Ik-Bu SOHN ${ }^{* 3}$, Young-Chul NOH ${ }^{* 3}$, and Man Seop LEE ${ }^{* 4}$ \\ ${ }^{* 1}$ Electronics and Communication Engineering Discipline, School of Science, Engineering and \\ Technology, Khulna University, Khulna 9208, Bangladesh \\ ${ }^{* 2}$ R\&D Center, Samsung Corning Precision Materials, 1320-10 Seocho-dong, Seocho-gu, Seoul, \\ Republic of Korea. \\ *3 Advanced Photonics Research Institute (APRI), Gwangju Institute of Science and Technology \\ (GIST), 1 Oryong-dong, Buk-gu, Gwangju 500-712, Republic of Korea \\ ${ }^{* 4}$ Department of Electrical Engineering, Korea Advanced Institute of Science and Technology \\ (KAIST), 373-1 Guseong-dong, Yuseong-gu, Daejeon 305-701, Republic of Korea \\ E-mail:shamim@kaist.ac.kr
}

\begin{abstract}
This paper reports the formation of periodic micro/nano-holes on the surface of as well as inside boro-aluminosilicate glass by controlling the irradiation conditions of a single-beam femtosecond laser. The laser beam is focused inside the glass sample to fabricate closely spaced periodic high aspect ratio nano-scale voids' array. The lowest diameter of the nanovoids reaches down to $650 \mathrm{~nm}$. We also pattern periodic microholes' array on the surface of the sample glass by focusing the laser beam on the front surface of the glass substrate. The fabricated micro/nano-metric holes and voids are very uniform in size and shape throughout large sample area. We also investigate the surface properties of the nanovoids' evolved glass surface. We don't observe any significant variation in surface morphology and materials' composition among the bare glass and nanovoids' engraved glass surface. Moreover, we qualitatively explain the formation mechanism of high aspect ratio nanovoids inside the glass substrate.

DOI: 10.2961/jlmn.2014.01.0005
\end{abstract}

Keywords: Femtosecond laser, single beam direct laser writing, boro-aluminosilicate glass, microholes, nanovoids

\section{Introduction}

Initially developed in $1990 \mathrm{~s}$, the first generation of femtosecond lasers, based on titanium doped sapphire as the active material, has opened a new era to the researchers to take advantage of the ultra-short pulse duration and high optical power offered by femtosecond lasers. During last two decades, femtosecond lasers have proven their ability in micro/nano-machining of a large variety of materials [112]. In recent years, femtosecond lasers have been considered as a promising tool to pattern micro/nano-scale structures on the surface of and inside various materials [13-23]. A variety of femtosecond laser based technologies have been utilized to engineer micro/nano-scale patterns: nearfield enhancement by particle lens array (PLA) [13], holographic technology [14], parallel diffractive multi-beam technology [15], and single beam direct laser writing (SBDLW) technology [16-23]. PLA based technology requires additional micro/nano-scale particles on material surface for structuring materials. PLA based technology has limitation in fabricating structures inside any material. In addition to this limitation, holographic technology is limited to the diffraction pattern of the interfering beams. On the contrary, patterning materials by parallel diffractive multi-beam technique requires additional diffractive optical elements, which technology is also restricted to surface patterning. All of these technologies are somewhat complicated and require high precision. These limitations of other technologies bring SBDLW technology to the forefront of the research field. SBDLW technology is controllable and reliable to pattern periodic structures on the surface of and inside transparent materials without damaging the material surface. These advantages make SBDLW technique popular to the researchers.

Several research groups reported the formation of subwavelength range holes or voids in glass materials [16-23] using SBDLW technology. Ahsan et. al. reported the fabrication of periodic nano-scale holes/voids on the surface of and inside soda-lime glass using single pulse irradiation in each spot [16]. Single nanoholes of variable size were printed on the glass surface. The periodic nanovoids fabricated inside soda-lime glass show inconsistency in size and shape. Nanoholes were absent in some spots of the sample area. In addition, circular shape heat affected zone was visible around the nanovoids those hindered the reduction of nanovoids' period. Zhang et. al. demonstrated the formation of single nanoholes in chalcogenide glass [17]. Single nanoholes have also been reported on cover glass by Joglekar et. al. [18]. Glezer et. al. reported nanoholes patterning in selective positions of fused silica glass surface [19]. Periodic nanoholes have also been engineered on borosilicate glass surface [20]. All of these research works [16-20] are primarily focused on the formation of low aspect ratio nanoholes or nanovoids. Several high aspect ratio nanovoids of moderate period were formed inside fused 
silica glass using very high numerical aperture lens [21]. Cracks are evident around the voids. Another research group reported the formation of periodic high aspect ratio nanoholes of long period on fused silica glass surface using high numerical aperture focusing with negative longitudinal spherical aberration [22]. White color halos are evolved around these nanoholes. There is clear evidence that closely located nanoholes may damage the glass surface and the surrounding nanoholes [23].

Usually, nanoholes are fabricated by applying laser pulses of energy just above the threshold energy of the material. For different materials, the threshold energy is different. The interactions of femtosecond laser pulses with each and every material are distinct as well. These parameters control the lowest diameter of nanoholes in various materials. At low laser energy, there is a possibility of energy fluctuation that may hinder the formation of consistent nanoholes over a large area. This phenomenon may cause the absence of micro/nano-holes in some places of the nanoholes' array. The size and shape might be influenced as well. For large area fabrication, flatness of the sample surface and the translation stage is very important. As a result, it is still challenging to pattern closely positioned high aspect ratio holes on the surface of and inside glasses over a large sample area by applying femtosecond laser pulses through a low numerical aperture lens.

In this paper, we demonstrate femtosecond laser induced patterning of closely spaced high aspect ratio nanovoids' array inside boro-aluminosilicate glass. We also fabricate periodic microholes' array on the glass surface. A suitable control over the laser fluence leads us to pattern micro/nano-scale holes or voids of variable diameter. The holes and voids were patterned over a large sample area (49 $\mathrm{mm}^{2}$ ) using single pulse irradiation in each spot, where the laser pulses were passed through a $50 \times$ achromatic objective lens of low numerical aperture. We also analyze the surface morphology and material properties of the nanovoids' engraved glass surface. Furthermore, we investigate the formation mechanism of high aspect ratio nanovoids inside the glass substrate. We strongly believe that, our experimental results and thorough analysis will provide an in-depth knowledge in this field.

\section{Experimental Details}

During the experiments, we utilized an amplified Ti:sapphire femtosecond laser source (Cyber Laser Inc., IFRIT-LH-C031) operating at the central wavelength $(\lambda)$ of $786 \mathrm{~nm}$ at a pulse repetition rate of $1 \mathrm{kHz}$. The pulse width $(\tau)$ of the femtosecond laser pulses was $183 \mathrm{fs}$. We used alkaline earth boro-aluminosilicate glass samples (EAGLE $\mathrm{XG}$, Corning; refractive index: 1.508 ; thickness: $0.5 \pm 0.05$ $\mathrm{mm}$ ) in our experiments. To fabricate periodic microholes' array on the surface of boro-aluminosilicate glass, the laser beam was focused on the front surface of the glass samples at normal incidence through a $50 \times$ achromatic objective lens (Mitutoyo, M Plan Apo NIR), whose focal length is 17 $\mathrm{mm}$ and numerical aperture is 0.42 . The same lens was employed to form periodic nano-scale voids inside the glass samples by focusing the laser beam inside them. The glass samples were mounted on a 3-axis linear translation stage (Laser Physics), which has resolution of $100 \mathrm{~nm}$ in all directions. To produce linearly polarized laser beam of con- trollable power, we placed a variable attenuator with built in beam-splitter (Newport) in the optical path. The computer-controlled attenuator was used for coarse power adjustment of the laser beam. The function of the polarizing cube beam splitter, consists of a rotating quartz phase $\lambda / 2$ wave plate, is to transmit p-polarized light while reflect spolarized light in the perpendicular direction. Figure 1 illustrates the schematic diagram of the experimental setup.

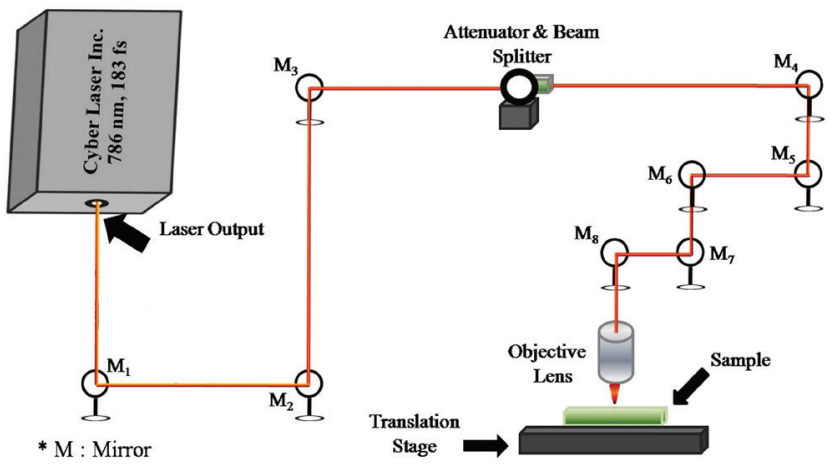

Figure 1. Schematic diagram of the experimental setup.

In order to analyze the surface morphology of the glass substrates, we used a field emission scanning electron microscope (FE-SEM) (JEOL Ltd., JSM-7401F), which is equipped with an energy dispersive spectrometer (EDS). The EDS analysis is suitable to identify the elemental composition of a sample. In order to take the side view of the nanovoids inside the glass samples using SEM, we broke the glass substrate vertically using conventional technique and polished the glass sample mechanically to expose the voids' encoded layer. Afterward, we rotated the sample by $90^{\circ}$ and placed inside the SEM so that we can examine the side view of the voids perpendicularly. Prior to SEM examination, the glass sample was sputter-coated with a thin layer of platinum to provide conductivity for SEM imaging An optical microscope (OM) was used to investigate the fabricated periodic micro/nano-scale holes and voids.

\section{Experimental Results}

By controlling the irradiation conditions of the laser beam, we fabricated periodic nano-scale voids' array inside boro-aluminosilicate glass. We also patterned microholes on the sample glass surface. The fabricated holes and voids show great homogeneity throughout $7 \mathrm{~mm} \times 7 \mathrm{~mm}$ sample area. The voids were fabricated inside different positions of the glass (depth varies from $5-17.25 \mu \mathrm{m}$ ). The diameter of the holes and voids was controlled by varying the laser fluence that ranges between $8.59-48.9 \mathrm{~J} / \mathrm{cm}^{2}$.

\subsection{Periodic nanovoids' array inside glass}

We have patterned periodic nanovoids of variable diameter and period at different depths inside boro-aluminosilicate glass. The diameter of the voids was ranging between 650 $\mathrm{nm}$ to $900 \mathrm{~nm}$. The period in the horizontal direction was varying between $1.15-1.75 \mu \mathrm{m}$ that was controlled by the scanning speed; whereas the period in the vertical direction was ranging between $2-5 \mu \mathrm{m}$ that was controlled by varying the scanning step. We also varied the focal point position of the laser beam from $5-17.25 \mu \mathrm{m}$ to pattern 


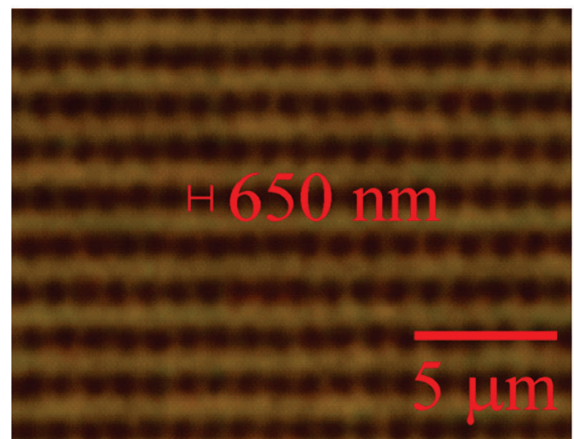

(a)

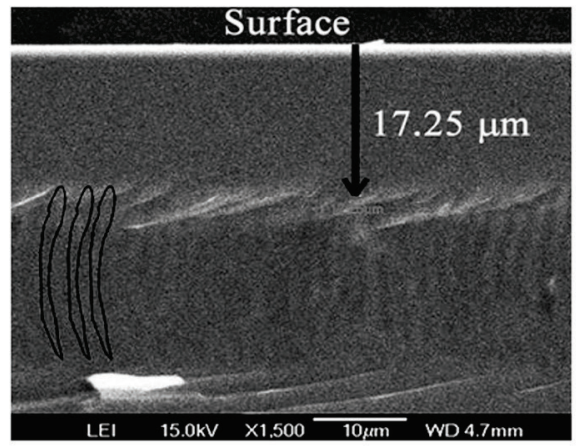

(d)

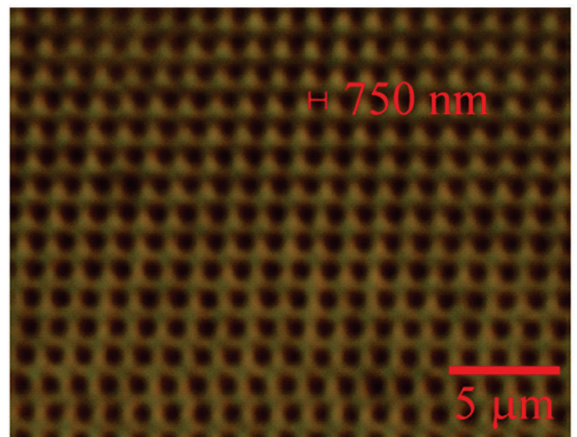

(b)

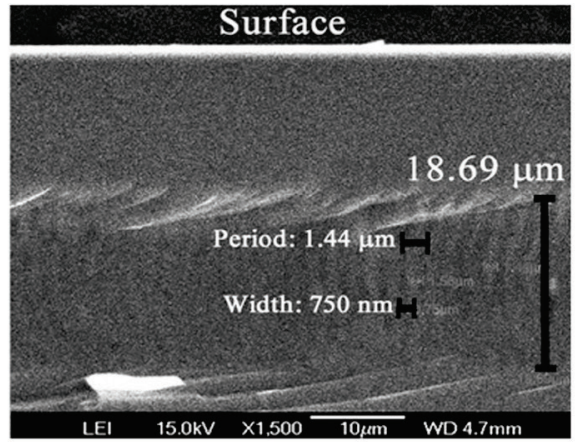

(e)

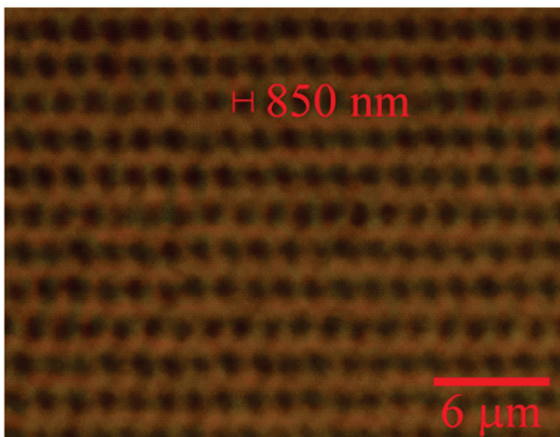

(c)

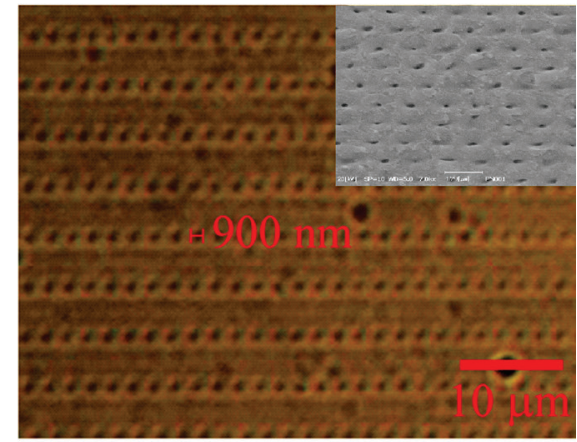

(f)

Figure 2. Images of the nanovoids' array formed inside boro-aluminosilicate glass. (a) OM image of the nanovoids of diameter $(D) 650$ $\mathrm{nm}$, fabricated at a depth $(d)$ of $10 \mu \mathrm{m}$ using laser fluence $(F)$ of $25.2 \mathrm{~J} / \mathrm{cm}^{2}$, horizontal period $\left(P_{H}\right)$ of $1.15 \mu \mathrm{m}$, and vertical period $\left(P_{V}\right)$ of $2 \mu \mathrm{m}$; (b) OM image of the nanovoids: $D=750 \mathrm{~nm}, d=10 \mu \mathrm{m}, F=39.63 \mathrm{~J} / \mathrm{cm}^{2}, P_{H}=1.35 \mu \mathrm{m}, P_{V}=2 \mu \mathrm{m}$; (c) OM image of the nanovoids: $D=850 \mathrm{~nm}, d=17.25 \mu \mathrm{m}, F=48.9 \mathrm{~J} / \mathrm{cm}^{2}, P_{H}=1.55 \mu \mathrm{m}, P_{V}=2 \mu \mathrm{m}$; (d-e) SEM image (side view) for the nanovoids of (c); (f) OM image of the nanovoids: $D=900 \mathrm{~nm}, d=5 \mu \mathrm{m}, F=20.05 \mathrm{~J} / \mathrm{cm}^{2}, P_{H}=1.75 \mu \mathrm{m}, P_{V}=5 \mu \mathrm{m}$ (SEM image of the glass surface is tagged with the OM image).

voids in different locations inside the glass substrates. The fabricated voids are regular in size and shape throughout the sample area, which are evident from Figure 2. The nanovoids of Figures 2(a) and 2(b) have been fabricated $10 \mu \mathrm{m}$ inside the glass substrates at a laser fluence of 25.2 and $39.63 \mathrm{~J} / \mathrm{cm}^{2}$, where the diameter of the nanovoids are $650 \mathrm{~nm}$ and $750 \mathrm{~nm}$, respectively. The scanning speed was $1.15 \mathrm{~mm} / \mathrm{s}$ for the nanovoids of Figure 2(a) and $1.35 \mathrm{~mm} / \mathrm{s}$ for the nanovoids of Figure 2(b).

Periodic nanovoids' array with voids' diameter of 850 $\mathrm{nm}$ have been printed $17.25 \mu \mathrm{m}$ inside glass at a laser fluence of $48.9 \mathrm{~J} / \mathrm{cm}^{2}$ and scanning speed of $1.55 \mathrm{~mm} / \mathrm{s}$, as shown in Figure 2(c). To investigate the configuration of the nanovoids of Figure 2(c), we broke the glass sample vertically. This process leads us to examine the side view of the nanovoids using SEM, which is illustrated in Figures 2(d) and 2(e). Surprisingly, the height of the nanovoids of Figure 2 (c) is very high $(\sim 18.69 \mu \mathrm{m})$, as illustrated in Figure 2(e). Although the diameter of the nanovoids is $850 \mathrm{~nm}$ on the front surface, the average value of diameter inside the glass sample is measured as $750 \mathrm{~nm}$. This result indicates that the nanovoids have high aspect ratio (aspect ratio $\sim 25$ ). Because of the complexity of the investigation technique, we limit the examination of voids' height to Figure 2(c) only. We expect similar kind of results for other nanovoids' of Figure 2.

We also fabricated periodic nanovoids of $900 \mathrm{~nm}$ diameter just $5 \mu \mathrm{m}$ below the glass surface at a scanning speed of $1.75 \mathrm{~mm} / \mathrm{s}$, the optical microscopic image of

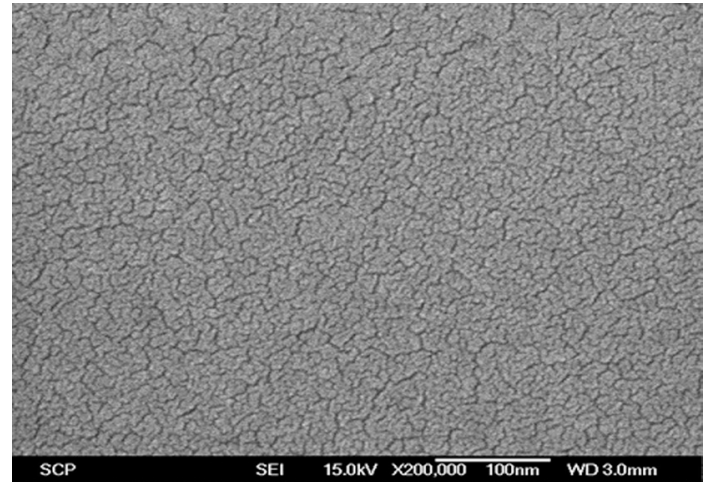

(a)

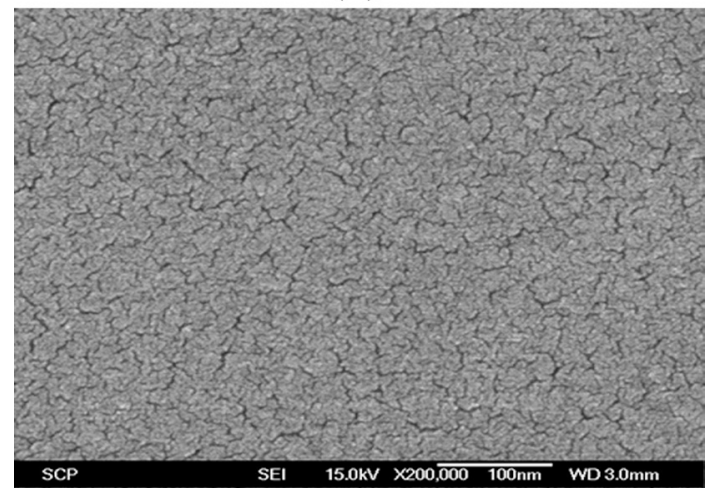

(b)

Figure 3. SEM images of the sample surfaces. (a) Bare glass; (b) nanovoids' engraved sample of Figure 2(c). 
which is shown in Figure 2(f). There is clear evidence of damage on this nanovoids' engraved glass surface that is obvious from the SEM image of the glass surface shown in the inset of Figure 2(f). We investigated all the nanovoids' inscribed glass surfaces. Unlike the sample of Figure 2(f), we don't observe any damage or significant variation on other glass surfaces of Figures 2. Figure 3 illustrates the SEM images of a bare glass and the nanovoids' engraved sample of Figure 2(c). A close observation of Figure 3 indicates no significant changes on the nanovoid's imprinted sample surface. We also analyze the EDS spectrum for both these samples, which is represented in Figure 4. The presence of various compounds along with materials' composition of the bare glass and the nanovoids' evolved glass samples are almost alike.

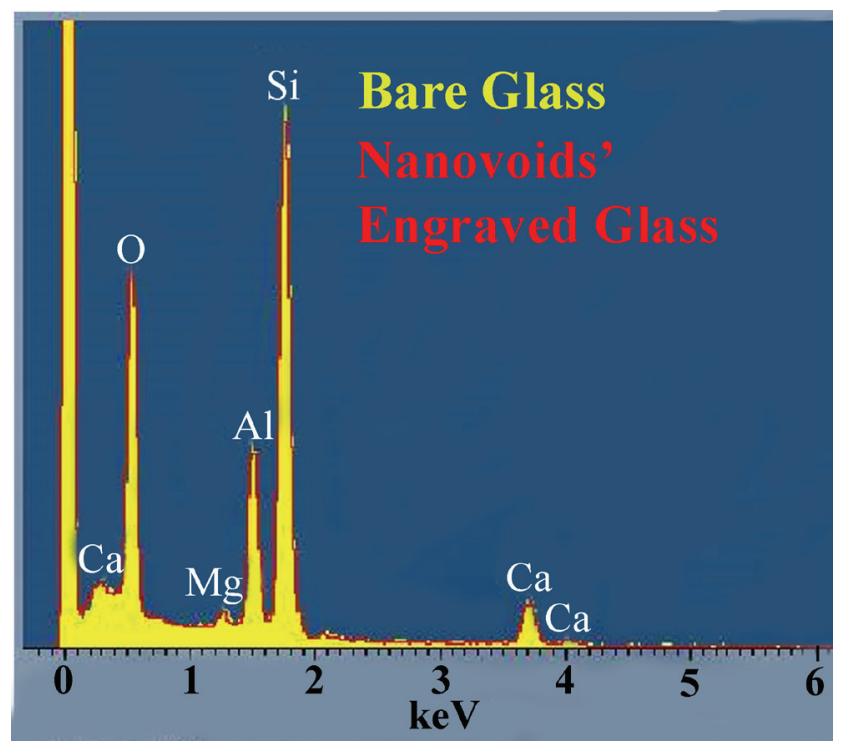

Figure 4. EDS spectrum for bare glass (yellow color) and nanovoids' engraved glass sample of Figure 2(c) (red color).

Table I summarizes the composition of the bare glass and nanovoids' inscribed boro-aluminosilicate glass sample of Figure 2(c). From Table I, it is obvious that the nanovoids' array fabricated $17.25 \mu \mathrm{m}$ inside the glass substrate have negligible influence in altering the material property of the sample glass. Similar characteristics are expected from the other voids' engraved samples of Figure 2, except the sample of Figure 2(f).

Table I

Composition of various boro-aluminosilicate glass substrates.

\begin{tabular}{ccc}
\hline Compounds & $\begin{array}{c}\text { Bare glass } \\
(\%)\end{array}$ & $\begin{array}{c}\text { After voids formation } \\
\text { (Figure 2(c) })(\%)\end{array}$ \\
\hline $\mathrm{MgO}$ & 1.4 & 1.2 \\
\hline $\mathrm{Al}_{2} \mathrm{O}_{3}$ & 18.5 & 18.4 \\
\hline $\mathrm{SiO}_{2}$ & 71.4 & 72.3 \\
\hline $\mathrm{CaO}$ & 8.7 & 8.1 \\
\hline
\end{tabular}

\subsection{Periodic microholes' array on glass surface}

We also fabricated periodic microholes' array on the surface of boro-aluminosilicate glass by varying the irradiation conditions of the femtosecond laser beam. Figure 5 illustrates the optical microscopic images of the microholes patterned on the glass surfaces. Figure 5(a) represents periodic microholes' array with holes' diameter of $1.3 \mu \mathrm{m}$. These microholes are printed on the sample glass substrate at a laser fluence of $8.59 \mathrm{~J} / \mathrm{cm}^{2}$. Microholes' array with holes' diameter of $1.5 \mu \mathrm{m}$ have also been patterned on the sample substrate at a laser fluence of $11.3 \mathrm{~J} / \mathrm{cm}^{2}$. In both cases, the microholes were fabricated at a scanning speed of $2 \mathrm{~mm} / \mathrm{s}$ and scanning step of $5 \mu \mathrm{m}$.

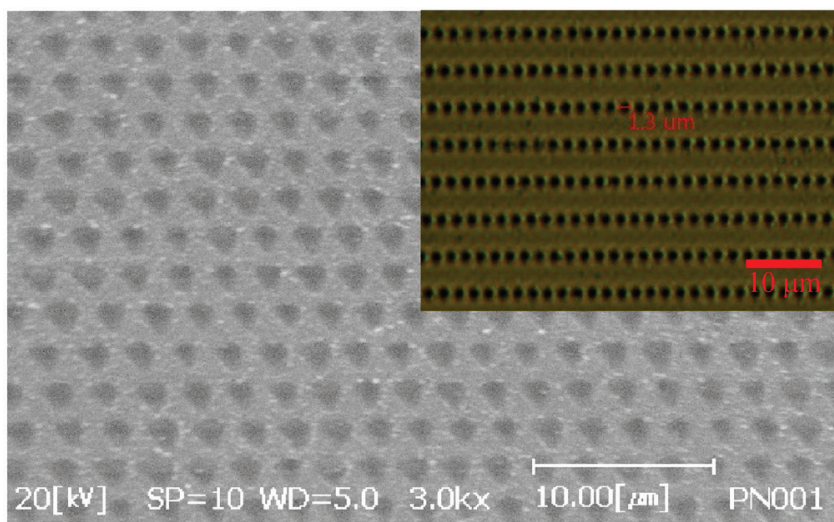

(a)

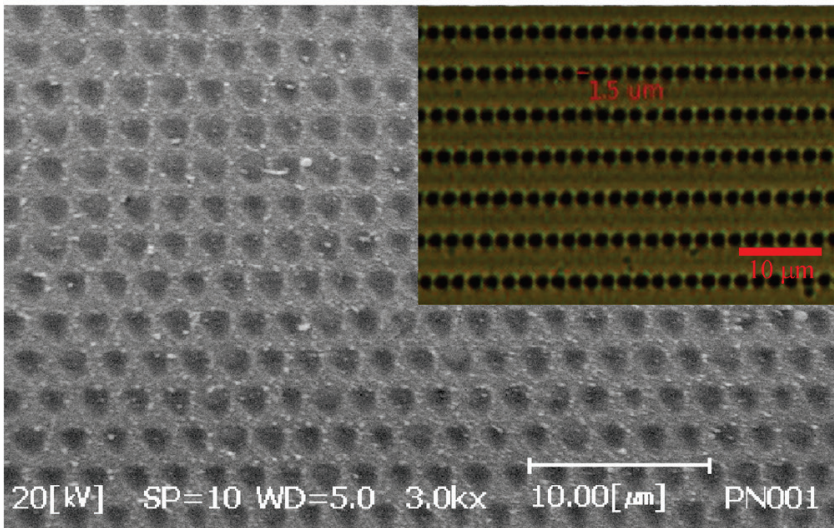

(b)

Figure 5. SEM images along with $\mathrm{OM}$ images of the periodic microholes' array on boro-aluminosilicate glass surface. (a) $D=$ $1.3 \mu \mathrm{m}, F=8.59 \mathrm{~J} / \mathrm{cm}^{2}, P_{H}=2 \mu \mathrm{m}, P_{V}=5 \mu \mathrm{m}$; (b) $D=1.5 \mu \mathrm{m}, F$ $=11.3 \mathrm{~J} / \mathrm{cm}^{2}, P_{H}=2 \mu \mathrm{m}, P_{V}=5 \mu \mathrm{m}$.

\section{Discussion}

This paper demonstrates the formation of closely positioned periodic micro/nano-holes' array on the surface of and inside boro-aluminosilicate glass substrates using low numerical aperture lens. The periodic micro/nano-scale holes and voids have consistent size and shape over a large sample area $\left(49 \mathrm{~mm}^{2}\right)$. As mentioned before, there have been reports of forming periodic nanovoids inside glasses in a small area. However, patterning nano-scale holes or voids of regular size and shape over a large sample area is critical. The variation of laser energy and inconsistent flatness of the samples \& the translation stage may affect the fabrication of uniform nanovoids or nanoholes over a large sample area. Besides, the heat affected zone around the holes/voids may inhibit the reduction of period of the holes or voids. As a result, closely positioned nano-scale holes/voids are difficult to fabricate without damaging surrounding structures [23]. A suitable control over the laser 
parameters make it possible to pattern closely spaced nanovoids' array of uniform size and shape throughout a large sample area inside the glass samples. We also investigate that the laser fluence required for fabricating periodic nanovoids of Figures 2(a-c), and 2(f) is higher than the laser fluence required for forming microholes' array of Figure 5. These results indicate a significant energy loss inside the glass substrates. The threshold energy required to pattern voids and holes depends on their location, which shows increasing trend with the increase of depth from the front surface of the glass samples. For a Gaussian laser beam, the radius of the focal spot size can be approximated by the following equation [24].

$$
w \approx \frac{\lambda f}{\pi w_{0}}
$$

where $\lambda$ is the wavelength of the incident light, $f$ is the focal length and $w_{0}$ is the $1 / \mathrm{e}$ Gaussian beam radius. In our case, $\lambda=786 \mathrm{~nm}, f=17 \mathrm{~mm}$, and $w_{0}=2.3 \mathrm{~mm}$. Thus the focal line width of the laser beam results in $2 w \approx 3.7 \mu \mathrm{m}$. Due to the reduction of the laser energy, only the central part of the Gaussian laser beam crosses the threshold energy that enables us to imprint micro/nano-metric holes/voids with diameter lower than the theoretical focal spot size. The confocal parameter (b) of the Gaussian laser beam can be obtained from the following Rayleigh range $\left(z_{\mathrm{R}}\right)$ equation in free space [25].

$$
b=2 z_{\mathrm{R}}=2 \frac{\pi w^{2}}{\lambda}
$$

Here the waist of the beam at the focal spot $(w)$ is equal to the radius of the spot size obtained from Eq. (1), i.e., 1.85 $\mu \mathrm{m}$. Thus, the confocal parameter $\mathrm{b} \approx 27.35 \mu \mathrm{m}$.

Most of the nanovoids reported in glass materials have low aspect ratio, especially when low numerical aperture objective lenses are used for patterning. It is still challenging to form high-aspect ratio nanovoids or nanoholes in glass materials using low numerical aperture lenses. We used a $50 \times$ achromatic objective lens of low numerical aperture $(0.42)$ to fabricate the nanovoids. The nanovoids of Figure 2(c), imprinted $17.25 \mu \mathrm{m}$ inside the glass substrate, have high aspect ratio that is approximately equal to 25 (height: $18.69 \mu \mathrm{m}$, diameter: $750 \mathrm{~nm}$ ). This result is obvious from the SEM image of Figure 2(e). Due to the complexity of the measurement technology, we considered only one sample (sample of Figure 2(c)) for SEM examination inside the glass sample. We utilized same technology to pattern nanovoids inside other boro-aluminosilicate glass samples. Thus we can infer that, those nanovoids also have high aspect ratio.

The fabrication of high aspect ratio periodic nanovoids' array was possible due to the involvement of several factors: formation of filaments due to the Kerr effect of the glass material, spherical aberration of the objective lens, chromatic aberration of the objective lens, and chromatic dispersion of glass sample. High intense femtosecond laser beam can create electron plasma filaments in transparent materials, which can extend the focal point of the laser beam. Alternate self-focusing and defocusing of electron plasma can guide a laser beam through a prolonged narrow channel, also known as filamentation [26]. The critical power for self-focusing can be obtained by the following equation [27].

$$
P_{c}=\frac{3.77 \lambda^{2}}{8 \pi n n_{2}}
$$

where $n$ is the linear and $n_{2}$ is the non-linear terms of refractive index of the medium. For boro-aluminosilicate glass sample, the critical power of self-focusing $P_{c} \approx 1.7$ MW for $\lambda=786 \mathrm{~nm}$. For a pulse width of $183 \mathrm{fs}$, the critical energy for self-focusing is calculated as $E_{c}=311 \mathrm{~nJ}$. The energy of the applied laser pulses during our experiments were in the micro-joule regime (varying from $0.36-1.24 \mu \mathrm{J}$ ) and therefore far beyond the critical-energy for selffocusing. Although there was energy loss when the voids were fabricated inside glass, still the applied energy was high enough for self-focusing.

Spherical aberration of the objective lens can also influence the fabrication of high aspect ratio voids inside transparent materials. An objective lens, suffering from spherical aberration effect, focuses optical rays more tightly if they inter far from the optical axis than if they enter closer to the optical axis. Although the effect caused by spherical aberration is minimal at the material surface, but this effect may cause the lengthening of focal spot inside glass materials. Furthermore, chromatic aberration can also prolong the focal point of the laser beam. Usually, lenses have different refractive index values for different wavelengths of light. The refractive index shows decreasing trend with the increase of wavelength. As a result, different colors focus at different positions on the optical axis of the lens inside glass substrates. These effects may cause the formation of high aspect ratio voids inside our glass samples. However, during our experiments we used apochromatic objective lens that has better correction of chromatic and spherical aberration. As a result, chromatic aberration and spherical aberration effects are minimized but still have some effects on the voids' height.

Chromatic dispersion of the sample glasses ( $\left.d n / d \lambda=-0.0385 \mu \mathrm{m}^{-1}\right)$ might have significant influence on the formation of high aspect ratio nanovoids. The material dispersion effect may arise from a frequency dependent response of the glass samples to incident laser beam, which may spread different frequencies of light inside the glass material. The group velocity is a function of the frequency of the incident light that causes group velocity dispersion (GVD). This phenomenon affects a short pulse of light to spread in time because of different frequency components of the pulse traveling at different velocities. GVD can be classified by the following group delay dispersion parameter $(D)$, where $c$ is the speed of light. [28].

$$
D=-\frac{\lambda}{c} \frac{d^{2} n}{d \lambda^{2}}
$$

As a result, the focal point of the laser beam is expected to be elongated. Aggregation of all these effects influenced the formation of high aspect ratio nanovoids inside boroaluminosilicate glass samples, especially when they are fabricated far below the glass surface.

If we closely examine the glass surfaces of Figure 2, we don't find any significant changes or damage on the nanovoids engraved glass surfaces, except for the glass sample of Figure 2(e). The damage in Figure 2(f) is occurred because of the short distance from the material surface $(5 \mu \mathrm{m})$. 
However, when nanovoids have been fabricated far from the front surface (starting from $7 \mu \mathrm{m}$ ) no damage is observed on the glass surfaces. The EDS spectrum of Figure 4 indicates that there is no major variation on the materials' composition in the nanovoids engraved glass surface of Figure 2(c), printed $17.25 \mu \mathrm{m}$ inside the glass sample. There is no absolute value of distance from the front surface below which we can offer damage-less patterning, but this distance increases with the applied laser energy. Spherical and chromatic aberration effects of the objective lens along with chromatic dispersion of the material might have influenced the damaging of the material surface. The experimental results indicate that, by properly controlling the laser energy we can avoid damage or variation of compounds on the structured glass surface.

\section{Conclusion}

In summary, we patterned closely spaced array of high aspect ratio nanovoids inside boro-aluminosilicate glass samples. We also fabricated microholes' array on the glass surfaces. Furthermore, we investigated the surface morphology and materials' composition for the nanovoids' engraved glass substrates. No damage was observed on the glass surfaces, when the nanovoids were fabricated more than $7 \mu \mathrm{m}$ inside the glass substrates. There was no significant changes in the composition of various compounds in the nanovoids' evolved glass samples. This result indicates that nanovoids can be patterned inside glass substrates without modifying materials' property. We also investigated the formation mechanism of the high aspect ratio voids and discovered several affects responsible for this phenomenon: filamentation, spherical \& chromatic aberration of the objective lens, and chromatic dispersion of the material. Although our results provide an in-depth knowledge in the field, we are unable to examine the SEM images inside all the glass samples due to technical difficulty. As a result, more investigation is required in the field to find an appropriate relationship among the voids' height, depth, and laser fluence. We strongly believe that, the periodic holes and voids will have diverse industrial applications in the field of optics and photonics, especially for the enhancement of light absorption and light spreading.

\section{Acknowledgments}

We are thankful to Prof. Mihail Lucian Pascu, National Institute for Laser, Plasma and Radiation Physics, Romania and Dr. Michael Shirk, Raydience, Inc., USA for their valuable information regarding spherical and chromatic aberration effect of objective lenses.

\section{References}

[1] R. Suo, J. Lousteau, H. Li, X. Jiang, K. Zhou, L. Zhang, W. N. MacPherson, H. T. Bookey, J. S. Barton, A. K. Kar, A. Jha and I. Bennion: Opt. Express, 17, (2009) 7540.

[2] M. S. Ahsan, F. Ahmed, Y. G. Kim, M. S. Lee and M. B. G. Jun: Appl. Surf. Sci., 257, (2011) 7771.

[3] C. W. Weng, W. C. Shen, C. Y. Lin, Y. J. Lee and J. S. Chen: Appl. Phys. A, 101, (2010) 243.

[4] M. S. Ahsan, F. Dewanda, M. S. Lee, H. Sekita and T. Sumiyoshi: Appl. Surf. Sci., 265, (2013) 784.
[5] C. Hnatovsky, E. Simova, P. P. Rajeev, D. M. Rayner, P. B. Corkum and R. S. Taylor: Opt. Lett., 32, (2007) 1459.

[6] M. S. Ahsan and M. S. Lee: Opt. Eng., 51, (2012) 121815.

[7] V. Maselli, J. R. Grenier, S. Ho and P. R. Herman: Opt. Express, 17, (2009) 11719.

[8] M. S. Ahsan, Y. G. Kim and M. S. Lee: J. Laser Micro Nanoen., 7, (2012) 164.

[9] G. D. Valle, R. Osellame and P. Laporta: J. Opt. A, 11, (2009) 013001.

[10] M. S. Ahsan and M. S. Lee: Optik, (2013)

[11] Y. Huang, S. Liu, W. Li, Y. Liu and W. Yang: Opt. Express, 17, (2009) 20756.

[12] J. Kim, W. Ha, J. Park, J. K. Kim, I. -B. Sohn, W. Shin and K. Oh: IEEE Photon. Technol. Lett., 25, (2013) 761.

[13] Y. Zhou, M. H. Hong, J. Y. H. Fuh, L. Lu, B. S. Lukyanchuk and Z. B. Wang: J. Alloy Compd., 449 (2008) 246.

[14] M. Hirano, K. -I. Kawamura and H. Hosono: Appl. Surf. Sci., 197-198, (2002) 688.

[15]Z. Kuang, D. Liu, W. Perrie, S. Edwardson, M. Sharp, E. Fearon, G. Dearden and K. Watkins, Appl. Surf. Sci, 255, (2009) 6582.

[16] M. S. Ahsan, Y. G. Kim and M. S. Lee: J. NonCryst. Solids, 357, (2011) 851.

[17] Q. Zhang, H. Lin, B. Jia, L. Xu and M. Gu: Opt. Express, 18, (2010) 6885.

[18] A. P. Joglekar, H. Liu, G. J. Spooner, E. Meyhöfer, G. Mourou and A. J. Hunt: Appl. Phys. B, 77, (2003) 25.

[19] E. N. Glezer, M. Milosavljevic, L. Huang, R. J. Finlay, T. -H. Her, J. P. Callan and E. Mazur: Opt. Lett., 21, (1996) 2023.

[20]B. Delobelle, F. Courvoisier and P. Delobelle: Opt. Laser Eng., 48, (2010) 616.

[21]E. Toratani, M. Kamata and M. Obara: Appl. Phys. Lett., 87, (2005) 171103.

[22] Y. V. White, X. Li, Z. Sikorski, L. M. Davis and W. Hofmeister: Opt. Express, 16, (2008) 14411.

[23] M. S. Ahsan and B. M. R. Haque: J. Nanosci. Nanoeng. Appl., 2, (2012) 39.

[24]D. Grobnic, S. J. Mihailov, C. W. Smelser and H. Ding: IEEE Photon. Technol. Lett., 16, (2004) 2505.

[25] G. Gbur and E. Wolf: J. Mod. Opt., 48, (2001) 1735.

[26] M. S. Ahsan and M. S. Lee: "Femtosecond Laser Processing of Materials: Fundamentals, Technologies, and Applications" (LAP Lambert Academic Publishing, Saarbrücken, 2013) p.63.

[27]D. Esser, S. Rezaei, J. Li, P. R. Herman and J. Gottmann: Opt. Express, 19, (2011) 25632.

[28] D. R. Anderson, L. Johnson and F. G. Bell: “Troubleshooting Optical-fiber Networks" (Elsevier, California, 2004) p.366

(Received: July 15, 2013, Accepted: December 24, 2013) 